\title{
An Unusual Case of Paraspinal Schwannoma Causing Undiagnosed Low Back Pain
}

\section{ismail Kaya, $M D^{*}$}

Department of Neurosurgery, Ministry of Health, Şırnak State Hospital, Republic of Turkey

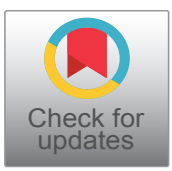

*Corresponding author: ismail KAYA (MD), Department of Neurosurgery, Ministry of Health, Şırnak State Hospital, Republic of Turkey, Tel: +905-469-257-340

\begin{abstract}
Paraspinal schwannomas are relatively common but hardly classified subject of spinal neurosurgery. We present a rare and unclassified case of paravertebral schwannoma that causes non-discogenic low back pain. A 45-year-old Caucasian female came to our clinic with low back pain. Her pain steadily worsened for two years. Her physical exam showed right straight leg raise positivity at 45-degree angle but provocation test negative. Magnetic resonance imaging showed a mass in the paravertebral muscle originating right lumbar 3-4 facet joint.

According to planned surgery patient operated. Histopathological diagnosis confirmed as schwannoma. The patient followed for 6 months without pain and neurologic deficiency. Follow up magnetic resonance imaging showed no residue or recurrence of the mass. Schwannomas must be taken into account for differential diagnosis of unexplained non-discogenic low back pain. New classification systems must be offered based on widespread patient groups.
\end{abstract}

\section{Keywords}

Paraspinal schwannomas, Non-discogenic low back pain, Paraspinal schwannoma classification, Surgical removal

\section{Abbreviations \\ L: Lumbar; \%: Percent symbol}

\section{Introduction}

Paraspinal schwannomas are relatively common but hardly classified subject of spinal neurosurgery. Schwannomas are rare causes of non-discogenic low back pain. We present an unusual case of paravertebral schwannoma that causes non-discogenic low back pain.

\section{Case Report}

A 45-year-old Caucasian female came to our clinic with low back pain at her right flank. She didn't describe neurologic claudication. Her pain steadily worsened for two years. The patient applied other hospitals in the area, but they couldn't find a reason. Her complaints have been worsened for 2 months. The patient has no incontinence.

Her physical exam showed right straight leg raise test positivity at 45 -degree angle but provocation test negative.

Magnetic resonance myelogram showed no cerebrospinal fluid flow obstruction (Figure 1). Careful evaluation of $\mathrm{T} 1$ weighted images showed a hypointense contrast-enhancing mass in the paravertebral muscle originating from the right lumbar (L) 3-4 facet joint (Figure 2). T2 weighted images showed a hyperintense mass in the same region (Figure 3).

We suspected paravertebral schwannoma. According to planned surgery patient went under general anesthesia. The patient carefully placed a tilting operating table and positioned as genupectorally. We used scopy to identify the surgical area. After we made adequate cleaning and closure identified skin and subcutaneous tissue passed via 2 centimeters of a paravertebral horizontal skin incision. Right paravertebral muscles dissected and lesion found. Lesion dissected until the right L3-4 facet joint. Lesion totally removed adhering to microsurgery principles. We did not harm the root with a top-down approach. Routinely curetted bony margins. Hemostasis provided. Layers closed according to anatomical orientation.

Histopathological diagnosis confirmed as schwannoma. 


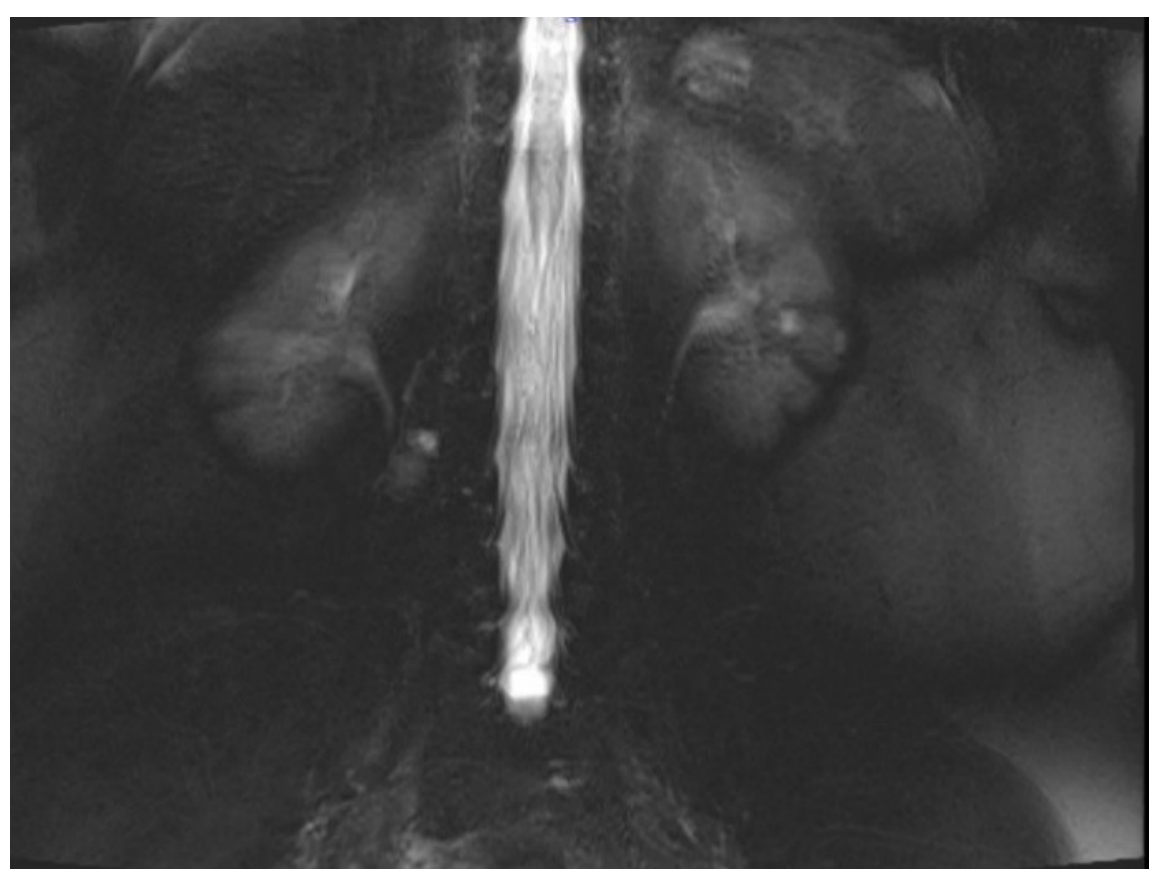

Figure 1: Magnetic resonance myelogram.

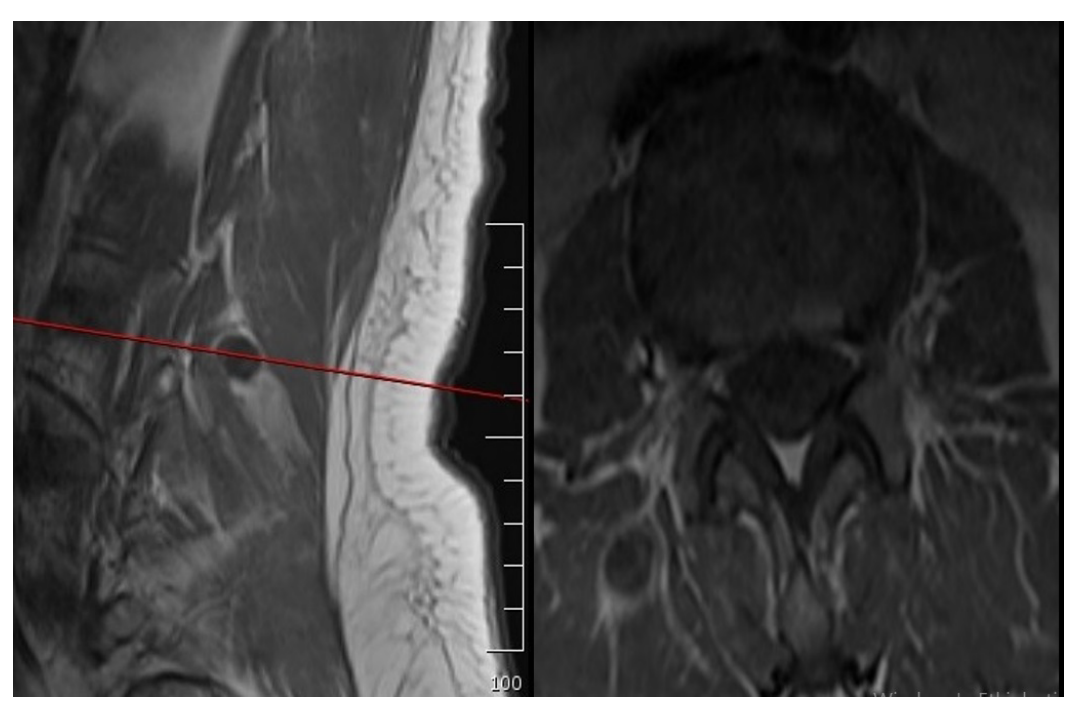

Figure 2: T1-weighted sagittal and axial magnetic resonance images.

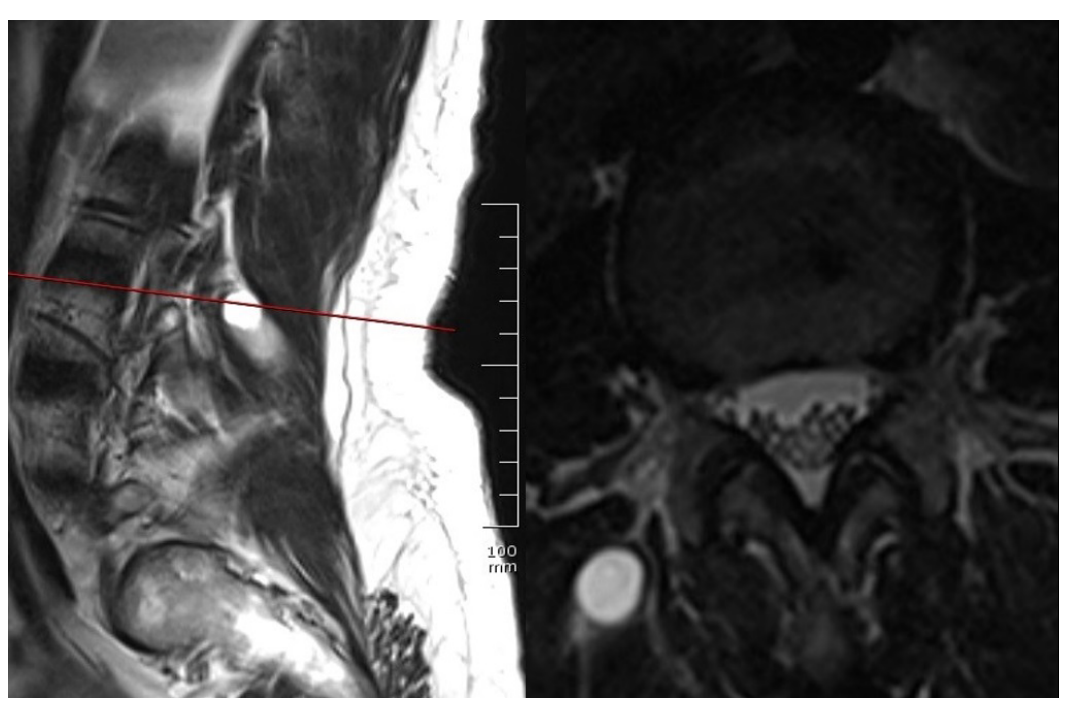

Figure 3: T2-weighted sagittal and axial magnetic resonance images. 


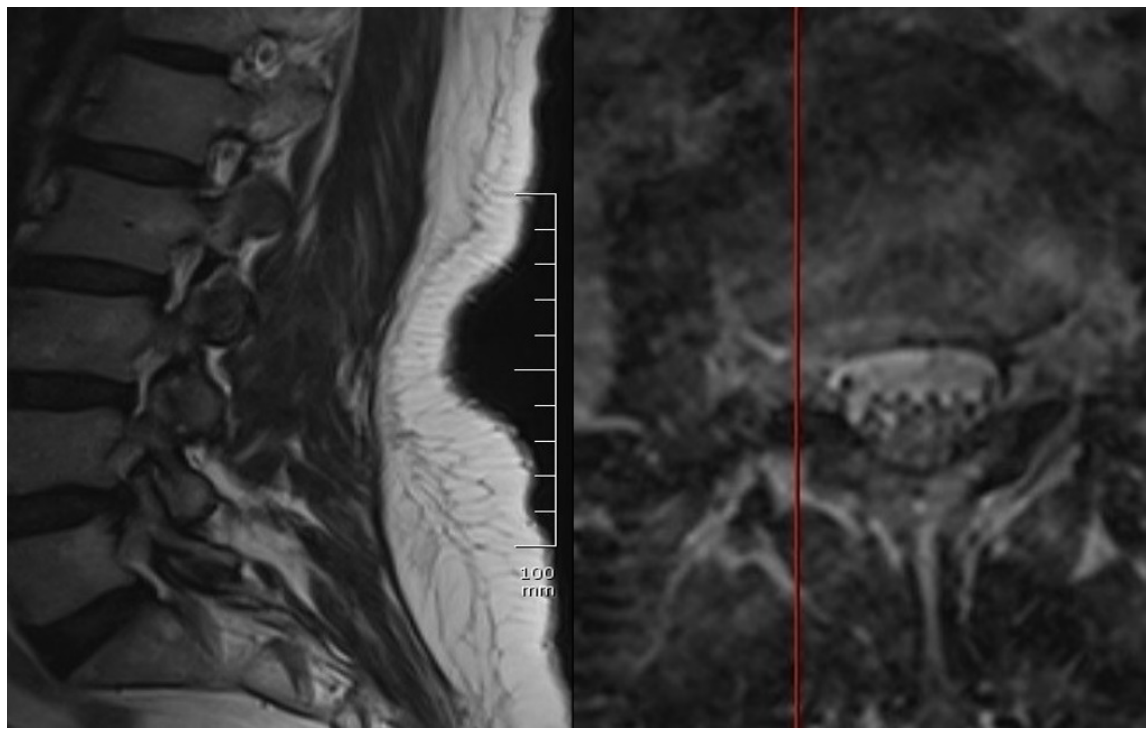

Figure 4: Follow up T2-weighted sagittal and axial magnetic resonance images.

The patient followed for 6 months without pain and neurologic deficiency. Follow up magnetic resonance scan showed no residue or recurrence of the mass (Figure 4).

\section{Discussion}

This case highlights one of the rare spinal causes of low back pain. Schwannomas are the most common peripheral nerve sheath tumors. It's originated from the Schwann cells. Schwannomas are also called neurilemmomas, usually seen between 30 and 60 years of age.

Radiologically although neurofibromas and schwannomas can look identical, schwannomas are frequently associated with hemorrhage, intrinsic vascular changes (thrombosis, sinusoidal dilatation), cyst formation and fatty degeneration. These findings are rare in neurofibromas. Signal characteristics at T1 weighted images $75 \%$ are isointense, $25 \%$ are hypointense; $\mathrm{T} 2$ weighted images more than $95 \%$ are hyperintense, often with mixed-signal; T1 weighted contrast-enhanced images virtually $100 \%$ enhances. Due to the structure of the cases that cannot be classified precisely and their similarity to surrounding tissues, magnetic resonance images should be examined carefully. In the present case, although the patient applied to other clinics for a long time, she was undiagnosed due to lack of imaging compatible with the herniated disc clinic. In our case, the lesion was noticed in the axial images with careful observation. We have found magnetic resonance imaging findings sufficient to explain complaints, as it is compatible with the examination. Therefore, no further investigation was requested.

Surgery provides good results with a very low rate of recurrence. Although the surgical method we used was known for muscle spasm after surgery, thanks to the microsurgical methods we used and the blunt dissection of the muscles, only the muscle spasm remained at the end of the first month and the patient recovered completely after the $6^{\text {th }}$ month. Even though malignant transformation has been described before it's very rare $[1,2]$. Postoperative neurological deficits are rare (less than $15 \%$ ) it can last for months. A neurological deficit can be caused by the contusion of the remaining continuous nerve fibers [3-5].

There are proposed classification systems but none of them covers all of the types $[6,7]$. Our case presents one of the unclassified paravertebral schwannoma cases.

\section{Conclusion}

Schwannomas must take account for differential diagnosis of unexplained non-discogenic low back pain. New classification systems must be offered based on widespread patient groups.

\section{Acknowledgements}

Special thanks for my bellowed wife Damla KAYA unquestionable support.

\section{Conflict of Interest}

The author doesn't have any potential conflicts of interest. This research did not receive any specific grant from funding agencies in the public, commercial, or not-for-profit sectors. This paper has been summarized as an electronic poster at the $31^{\text {st }}$ Scientific Congress of the Turkish Neurosurgical Society.

\section{Highlights}

- Schwannomas must be considered for differential diagnosis of low back pain.

- Surgery provides good results with a very low rate of recurrence.

- New classification systems must be offered for schwannomas. 


\section{References}

1. Khoshyomn S, Barth KN, Christman RA, Braff SP, Wilson JT (2002) Torsion of a lumbar nerve root schwannoma. Pediatr Neurosurg 37: 206-209.

2. Lahat E, Rothman AS, Aron AM (1986) Schwannoma presenting as lumbar disc disease in an adolescent boy. Ann Neurol 20: 643-644.

3. Lu DC, Dhall SS, Mummaneni PV (2009) Mini-open removal of extradural foraminal tumors of the lumbarspine. $J$ Neurosurg Spine 10: 46-50.

4. Russell SM (2007) Preserve the nerve: Microsurgical re- section of peripheral nerve sheath tumors. Neurosurgery 61: 113-118.

5. Rustagi T, Badve S, Parekh AN (2012) Sciatica from a foraminal lumbar root schwannoma: Case report and review of literature. Case Rep Orthop 2012: 142-143.

6. Sridhar K, Ramamurthi R, Vasudevan MC, Ramamurthi B (2001) Giant invasive spinal schwannomas: Definition and surgical management. J Neurosurg 210-215.

7. Park SC, Chung SK, Choe G, Kim HJ (2009) Spinal intraosseous schwannoma: A case report and review. J Korean Neurosurg Soc 46: 403-408. 